\title{
Hadronic transitions in bottomonium at Belle
}

\section{Elisa Guido*†}

INFN Sezione di Torino, Via Pietro Giuria 1, I-10125, Torino, Italy

E-mail: elisa.guido@to.infn.it

\begin{abstract}
The study of hadronic transitions among bottomonium states, and their relative magnitude, can be used as a test bench for non-perturbative approaches to QCD. In particular, transitions through an $\eta$ meson, despite involving a heavy quark spin symmetry violation, have been measured to have an unexpectedly enhanced branching fraction with respect to those via a dipion system. A set of recent results obtained using the data collected by the Belle experiment at the energy of the $\Upsilon(4 S)$ and $\Upsilon(5 S)$ resonances are presented. They include the measurement of the transition $\Upsilon(5 S) \rightarrow$ $\eta \Upsilon(1 D)$, an updated measurement of the branching fractions of $\Upsilon(4 S) \rightarrow \eta \Upsilon(1 S)$ and $\Upsilon(4 S) \rightarrow$ $\pi^{+} \pi^{-} \Upsilon(1 S, 2 S)$ decays, and searches for other possible transitions involving an $\eta$ meson.
\end{abstract}

XVII International Conference on Hadron Spectroscopy and Structure - Hadron2017 25-29 September, 2017

University of Salamanca, Salamanca, Spain

\footnotetext{
* Speaker.

${ }^{\dagger}$ On behalf of the Belle Collaboration.
} 
The last decade has been a fertile time for bottomonium spectroscopy, with the observation of many missing states and also of a few unexpected states, that can be classified as exotica. Spectroscopy and, more in general, a deep comprehension of the bottomonium system cannot prescind from the precision study of the transitions between its states. These transitions can be predicted by effective potential models, whose reliability is well established in the case of the dominant radiative transitions. Some puzzling features in the prediction of branching fractions are instead present in the description of hadronic transitions, in particular those involving an $\eta$ or a $\pi^{0}$ meson. Models based on QCD multipole expansion (QCDME) [1], where the expansion is performed in terms of momentum of the emitted pair of gluons, are expected to correctly describe the hadronic transitions. Processes involving gluonic 'M1' transitions, i.e. with a spin flip and therefore a heavy quark spin symmetry (HQSS) violation, are expected to be suppressed with respect to 'E1' transitions, that do not violate HQSS. Transitions like $\Upsilon(m S) \rightarrow \eta \Upsilon(n S)$ and $\Upsilon(m S) \rightarrow \pi^{+} \pi^{-} h_{b}(n P)$, with $m>n$, belong to the former case, while $\Upsilon(m S) \rightarrow \pi^{+} \pi^{-} \Upsilon(n S)$ are of the latter type. A further suppression is expected for transitions mediated by a $\pi^{0}$ meson, due to an additional isospin violation.

The experimental status of the measurements of bottomonium transitions via an $\eta$ meson from $\Upsilon(4 S, 3 S, 2 S)$ resonances is summarized in Table 1, while Table 2 lists the main measurements obtained in hadronic transitions of the $\Upsilon(5 S)$.

The first interesting and surprising result has been the measurement by BaBar [2] of a branching fraction $\mathscr{B}(\Upsilon(4 S) \rightarrow \eta \Upsilon(1 S))$ more than twice larger than the corresponding dipion transition $\left(\mathscr{B}\left(\Upsilon(4 S) \rightarrow \pi^{+} \pi^{-} \Upsilon(1 S)\right)=(0.81 \pm 0.06) \times 10^{-4}[6]\right)$. This result was in high tension with the QCDME models, predicting a suppression of the $\eta$-mediated transition, and prompted a vivid theoretical speculation aiming to explain the deviation (see [3, 4], for instance).

The result has been recently confirmed by Belle [5], which measured $\frac{\mathscr{B}(\Upsilon(4 S) \rightarrow \eta \Upsilon(1 S))}{\mathscr{B}\left(\Upsilon(4 S) \rightarrow \pi^{+} \pi^{-} \Upsilon(1 S)\right)}=$ $2.07 \pm 0.30$ (stat.) \pm 0.11 (syst.), by reconstructing $\eta \rightarrow \pi^{+} \pi^{-} \pi^{0}$ decays, with $\pi^{0} \rightarrow \gamma \gamma$, and the dimuon decays of $\Upsilon(1 S)$. The signal yields for the $\Upsilon(4 S) \rightarrow \eta \Upsilon(1 S)$ and $\Upsilon(4 S) \rightarrow \pi^{+} \pi^{-} \Upsilon(1 S)$ transitions are extracted by unbinned maximum likelihood fits to the distributions $\Delta M_{\eta}=M(\mu \mu \pi \pi \gamma \gamma)-$ $M(\mu \mu)-M(\pi \pi \gamma \gamma)$ and $\Delta M=M(\mu \mu \pi \pi)-M(\mu \mu)$, respectively, as shown in Fig. 1.
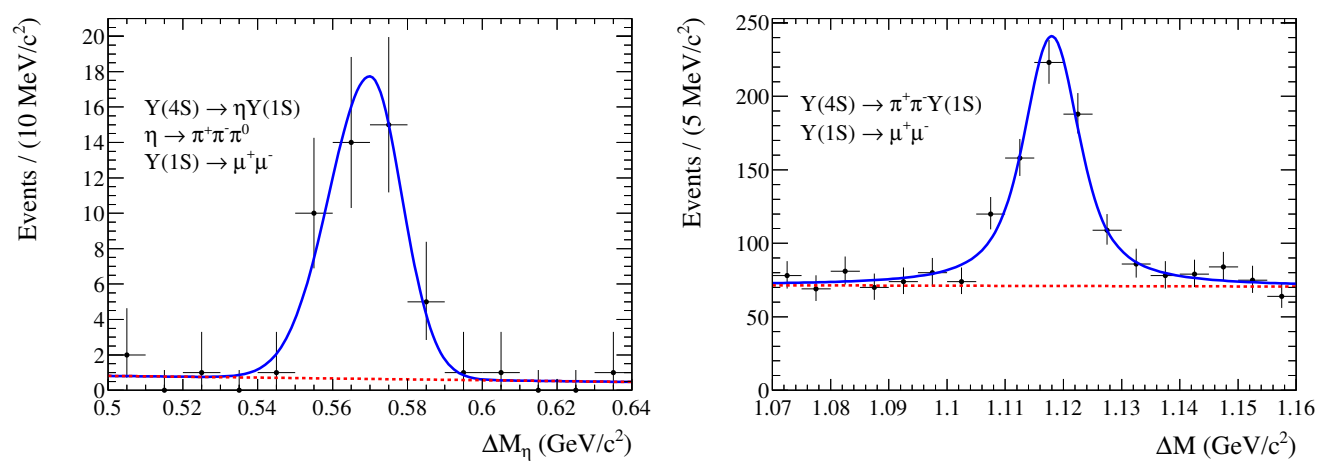

Figure 1: Fits as in [5] to the $\Delta M_{\eta}$ distribution for $\Upsilon(4 S) \rightarrow \eta \Upsilon(1 S)$ candidates (left), and to the $\Delta M$ distribution for $\Upsilon(4 S) \rightarrow \pi^{+} \pi^{-} \Upsilon(1 S)$ candidates (right). In each plot, data are shown as points, the solid blue line shows the best fit to the data, while the dashed red line shows the background contribution.

Following a similar approach, the transition $\Upsilon(1 D) \rightarrow \eta \Upsilon(1 S)$ is searched for. Such a transition is predicted to be enhanced with respect to the $\Upsilon(1 D) \rightarrow \pi^{+} \pi^{-} \Upsilon(1 S)$ transition by the axial 
anomaly in QCD [7]. The $\Upsilon(1 D)$ resonance could be produced via the yet unobserved doubly radiative transitions of $\Upsilon(4 S)$ through the $\chi_{b}(2 P)$ states $\left(\Upsilon(4 S) \rightarrow \gamma \chi_{b}(2 P), \chi_{b}(2 P) \rightarrow \gamma \Upsilon(1 D)\right)$. In the data sample analyzed, no significant signal has been found for such a process, and an upper limit (UL) on the product of branching fractions is set at the $90 \%$ of confidence level (CL): $\mathscr{B}(\Upsilon(4 S) \rightarrow \gamma \gamma \Upsilon(1 D)) \times \mathscr{B}(\Upsilon(1 D) \rightarrow \eta \Upsilon(1 S))<2.3 \times 10^{-5}[5]$.

In [5] a detailed study of dipion transitions in presented as well. Besides the already mentioned $\Upsilon(4 S) \rightarrow \pi^{+} \pi^{-} \Upsilon(1 S)$, the transitions $\Upsilon(4 S) \rightarrow \pi^{+} \pi^{-} \Upsilon(2 S)$ and $\Upsilon(2 S, 3 S) \rightarrow \pi^{+} \pi^{-} \Upsilon(1 S)$ with $\Upsilon(2 S, 3 S)$ produced in initial state radiation (ISR) events, are analyzed. The corresponding yields can be seen in Fig. 2 (left), that shows the two-dimensional distributions of $\Delta M$ vs. $M(\mu \mu)$ on the data. Improved values for the branching fractions of the transitions from $\Upsilon(4 S)$ are obtained, and the measured ISR-production cross sections for the $\Upsilon(2 S, 3 S)$ resonances, using the world-averaged values for $\mathscr{B}\left(\Upsilon(2 S, 3 S) \rightarrow \pi^{+} \pi^{-} \Upsilon(1 S)\right)$ [6], are found in agreement with the calculations [8].

For all the analyzed transitions, the distributions of the dipion invariant mass, $M\left(\pi^{+} \pi^{-}\right)$, and the helicity angle of the positive pion candidate, $\cos \theta_{\text {hel }}\left(\pi^{+}\right)$, are determined by unfolding the signal component from the data using the ${ }_{s} \mathscr{P}$ lot technique described in [9]. The major interest comes from the $M\left(\pi^{+} \pi^{-}\right)$distribution for the $\Upsilon(4 S) \rightarrow \pi^{+} \pi^{-} \Upsilon(1 S)$ transitions, that is displayed in Fig. 2 (right). The distribution shows an enhancement followed by a clear dip around $1 \mathrm{GeV} / \mathrm{c}^{2}$, likely due to a contribution from the $f_{0}(980)$ scalar meson and its interference with a non-resonant model. Such a pattern had not been seen in previous $\Upsilon(4 S)$ data [10,11], while a similar distribution has been observed in the dipion transitions from $\Upsilon$ resonances above the $B \bar{B}$ threshold [12,13], and has also been recently predicted by theory [14]. Data shows an indication, with $2.8 \sigma$ of statistical significance, of preferring a description that includes a resonant $\Upsilon(1 S) f_{0}(980)$ contribution on top of the non-resonant model. No improvement in the description is obtained by adding a resonant $\Upsilon(1 S) f_{2}(1270)$ component.
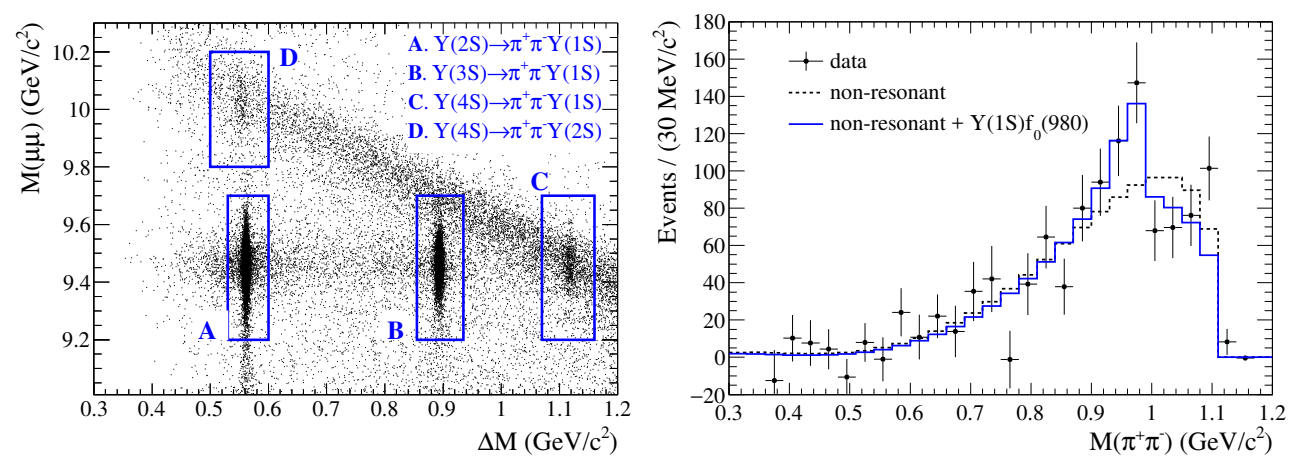

Figure 2: Distribution of $M(\mu \mu)$ vs. $\Delta M$ for the events selected on data as in [5]; fit regions for the four analyzed dipion transitions are enclosed in boxes (left). Fit as in [5] to the efficiency-corrected distribution of $M\left(\pi^{+} \pi^{-}\right)$for the signal component unfolded from the data distribution with the ${ }_{s} \mathscr{P}$ lot technique [9], in the $\Upsilon(4 S) \rightarrow \pi^{+} \pi^{-} \Upsilon(1 S)$ candidates (black points); the models used for the fit are: non-resonant model (black dashed line), and non-resonant $+\Upsilon(1 S) f_{0}(980)$ model (blue solid line) (right).

The enhanced $\Upsilon(4 S) \rightarrow \eta \Upsilon(1 S)$ rate is not the only contrast point between theory and experiment. Also for transitions from below-threshold resonances $(\Upsilon(2 S, 3 S))$ the branching fractions are not well predicted. In particular, $\mathscr{B}(\Upsilon(2 S) \rightarrow \eta \Upsilon(1 S))$ has been measured with compatible 
results by the CLEO [15], BaBar [16] and Belle [17] experiments to be smaller than the predicted value. The $\Upsilon(3 S) \rightarrow \eta \Upsilon(1 S)$ transition is still unobserved, with ULs on the branching fraction set by CLEO [15] and BaBar [16] that are already lower than the theoretically predicted value.

Another element to the picture is added by the observation of the transition $\Upsilon(4 S) \rightarrow \eta h_{b}(1 P)$ [18], that is to date the most probable measured non- $B \bar{B}$ decay of the $\Upsilon(4 S)$, with a branching fraction one order of magnitude larger than the other known hadronic transitions, and in agreement with the prediction [19].

\begin{tabular}{|l|c|c|c|c|c|c|}
\hline & \multicolumn{2}{|c|}{ CLEO } & \multicolumn{2}{c|}{ BaBar } & \multicolumn{2}{c|}{ Belle } \\
\hline Transition & $\mathscr{B}\left[10^{-4}\right]$ & Ref. & $\mathscr{B}\left[10^{-4}\right]$ & Ref. & $\mathscr{B}\left[10^{-4}\right]$ & Ref. \\
\hline$\Upsilon(4 S) \rightarrow \eta \Upsilon(1 S)$ & - & & $1.96 \pm 0.26 \pm 0.09$ & {$[2]$} & $1.70 \pm 0.23 \pm 0.08$ & {$[5]$} \\
$\Upsilon(3 S) \rightarrow \eta \Upsilon(1 S)$ & $<1.8$ & {$[15]$} & $<1.0$ & {$[16]$} & - & \\
$\Upsilon(2 S) \rightarrow \eta \Upsilon(1 S)$ & $2.1_{-0.6}^{+0.7} \pm 0.3$ & {$[15]$} & $2.39 \pm 0.31 \pm 0.14$ & {$[16]$} & $3.57 \pm 0.25 \pm 0.21$ & {$[17]$} \\
\hline$\Upsilon(4 S) \rightarrow \eta h_{b}(1 P)$ & - & & - & & $21.8 \pm 1.1 \pm 1.8$ & {$[18]$} \\
\hline
\end{tabular}

Table 1: Summary of measurements of the branching fractions of $\Upsilon(4 S, 3 S, 2 S)$ transitions to lower-mass bottomonia via an $\eta$ meson, obtained by the CLEO, BaBar and Belle experiments in the referenced works. The branching fractions are given in units of $10^{-4}$, the first error is statistical and the second systematic, while the ULs are at $90 \%$ of CL.

A further set of informative results is represented by the study of the $\Upsilon(5 S)$ transitions, performed by the Belle experiment, and summarized in Table 2. Dipion transitions from $\Upsilon(5 S)$ to both lower-mass $\Upsilon$ states [20] and $h_{b}(1 P, 2 P)$ states [21] proceed with enhanced rates, due to the $Z_{b}(10610)$ and $Z_{b}(10650)$ contributions. Despite such an enhancement, the corresponding $\eta$-mediated transitions are not suppressed [22,23].

\begin{tabular}{|l|c|c|c|c|}
\hline Transition & $\mathscr{B}\left({ }^{\prime} h '=\pi^{+} \pi^{-}\right)\left[10^{-3}\right]$ & Ref. & $\mathscr{B}\left({ }^{\prime} h '=\eta\right)\left[10^{-3}\right]$ & Ref. \\
\hline$\Upsilon(5 S) \rightarrow{ }^{\prime} h \Upsilon \Upsilon(1 S)$ & $5.3 \pm 0.6$ & {$[20]$} & $0.73 \pm 0.16 \pm 0.08$ & {$[22]$} \\
$\left.\Upsilon(5 S) \rightarrow{ }^{\prime} h\right\urcorner(2 S)$ & $7.8 \pm 1.3$ & {$[20]$} & $2.1 \pm 0.7 \pm 0.3$ & {$[23]$} \\
\hline$\Upsilon(5 S) \rightarrow{ }^{\prime} h ' h_{b}(1 P)$ & $3.5_{-1.3}^{+1.0}$ & {$[21]$} & $<3.3$ & {$[23]$} \\
$\Upsilon(5 S) \rightarrow{ }^{\prime} h ' h_{b}(2 P)$ & $5.7_{-2.1}^{+1.7}$ & {$[21]$} & $<3.7$ & {$[23]$} \\
\hline$\Upsilon(5 S) \rightarrow{ }^{\prime} h ' \Upsilon(1 D)$ & - & & $2.8 \pm 0.7 \pm 0.4$ & {$[23]$} \\
\hline
\end{tabular}

Table 2: Summary of measurements of the branching fractions of $\Upsilon(5 S)$ transitions to lower-mass bottomonia via a hadronic system ' $h$ ', given by a dipion or an $\eta$ meson, obtained by the Belle experiment in the references works. The branching fractions are given in units of $10^{-3}$, when two errors are shown the first one is statistical and the second systematic, while the ULs are at $90 \%$ of CL. Some results are preliminary.

In particular, Belle measured with a statistical significance of $4.4 \sigma$ the transition $\Upsilon(5 S) \rightarrow$ $\eta \Upsilon(1 D)$ [23], by reconstructing the $\eta \rightarrow \gamma \gamma$ decays and analyzing the $\eta$ missing mass spectrum after subtracting the contribution due to combinatorial background events. The measured branching fraction is compatible with a theoretical expectation [24], that, in the context of a rescattering model, describes the transition as proceeding via triangular $B^{(*)}$ meson loops. The experimental resolution does not allow to resolve the $\Upsilon(1 D)$ triplet yet, but an effort is made to investigate the potential sensitivity. The $\Upsilon(1 D)$ signal is parameterized as three Crystal Ball functions, with free relative fractions $f_{1}=\frac{\mathscr{B}\left(\Upsilon(5 S) \rightarrow \eta \Upsilon\left(1 D_{1}\right)\right)}{\mathscr{B}\left(\Upsilon(5 S) \rightarrow \eta \Upsilon\left(1 D_{2}\right)\right)}$ and $f_{3}=\frac{\mathscr{B}\left(\Upsilon(5 S) \rightarrow \eta \Upsilon\left(1 D_{2}\right)\right)}{\mathscr{B}\left(\Upsilon(5 S) \rightarrow \eta \Upsilon\left(1 D_{3}\right)\right)}$, and with the mass of $\Upsilon\left(1 D_{2}\right)$ 
fixed to its world-averaged value [6]. The signal extraction is performed in different configurations, with the mass splitting values of the $J=2$ state with the other two $\Upsilon(1 D)$ states, $\Delta M_{12}$ and $\Delta M_{23}$, chosen to range between $3 \mathrm{MeV} / \mathrm{c}^{2}$ and $15 \mathrm{MeV} / \mathrm{c}^{2}$, in agreement with calculations and previous observations. In all the cases, $f_{1}$ and $f_{3}$ assume values compatible with zero, and a scan of the $90 \%$ CL ULs on these fractions is performed as a function of $\Delta M_{12}$ and $\Delta M_{23}$, as shown in Fig. 3.
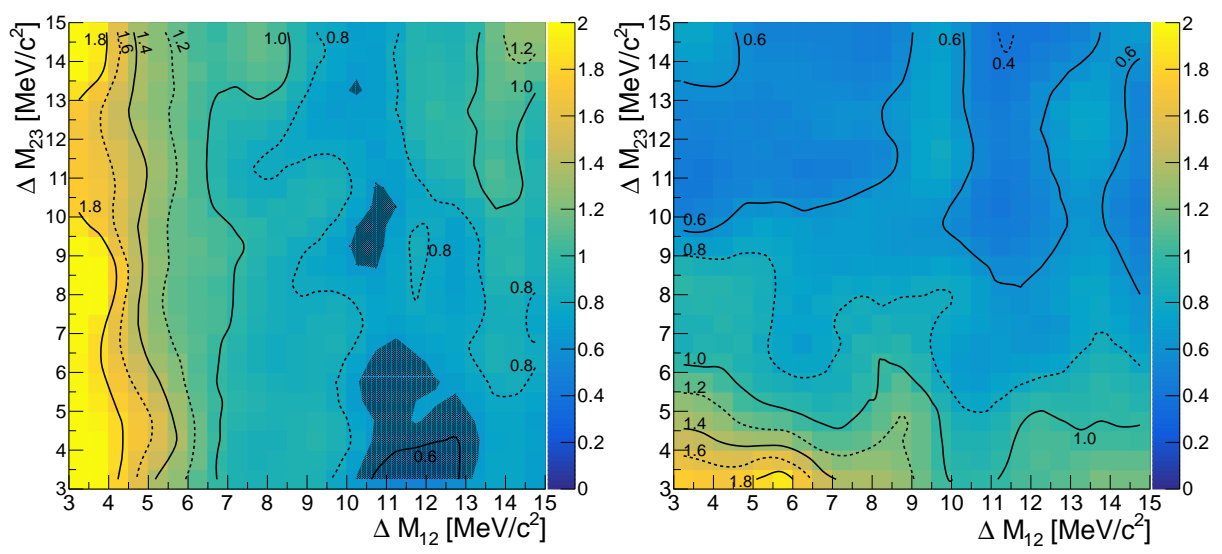

Figure 3: $90 \%$ CL ULs on $f_{1}$ (left) and $f_{3}$ (right), as function of the chosen mass splitting values $\Delta M_{12}$ and $\Delta M_{23}$. The black lines represent the curves at fixed values of the UL, from 0.4 to 1.8 in steps of 0.2. The corresponding UL value is reported next to each line. Dashed and solid line styles are alternated for clarity. The grey shaded areas correspond to the regions excluded by the theoretically-favored value $f_{1}=0.68$ [24]. The results are preliminary.

Hadronic transitions are a key ingredient in understanding bottomonium and, more in general, the QCD description of matter. The Belle experiment has recently given a solid contribution to the field, with:

- the observation of $\Upsilon(4 S) \rightarrow \eta h_{b}(1 P)[18]$;

- the measurement of $\Upsilon(5 S) \rightarrow \eta \Upsilon(1 D)$ [23], with a statistical significance close to $5 \sigma$;

- the confirmation of the enhancement of the HQSS-violating transition $\Upsilon(4 S) \rightarrow \eta \Upsilon(1 S)$ with respect to $\Upsilon(4 S) \rightarrow \pi^{+} \pi^{-} \Upsilon(1 S)[5]$;

- the measurement of or the search for other $\eta$-mediated transitions, such as $\Upsilon(1 D) \rightarrow \eta \Upsilon(1 S)$ or transitions from the $\Upsilon(5 S)$ to lower-mass bottomonia [5, 22, 23];

- the precision measurement of $\Upsilon(4 S)$ dipion transitions, with the first indication for a resonant contribution in $\Upsilon(4 S) \rightarrow \pi^{+} \pi^{-} \Upsilon(1 S)$ [5].

In the next future, the Belle II experiment at the Super KEKB asymmetric energy $e^{+} e^{-}$collider will be able to further investigate, on high-statistics data samples, the remaining open points in the study of bottomonium transitions. 


\section{References}

[1] Y. P. Kuang, Front. Phys. China 1, 19 (2006) and references therein.

[2] B. Aubert et al. [BaBar Collaboration], Phys. Rev. D 78, 112002 (2008).

[3] C. Meng and K. T. Chao, Phys. Rev. D 78, 074001 (2008).

[4] M. B. Voloshin, Mod. Phys. Lett. A 26, 773 (2011).

[5] E. Guido et al. [Belle Collaboration], Phys. Rev. D 96 no.5, 052005 (2017).

[6] C. Patrignani et al. [Particle Data Group], Chin. Phys. C, 40, 100001 (2016) and 2017 update.

[7] M. B. Voloshin, Phys. Lett. B 562, 68 (2003).

[8] M. Benayoun, S. I. Eidelman, V. N. Ivanchenko and Z. K. Silagadze, Mod. Phys. Lett. A 14, 2605 (1999) and references therein.

[9] M. Pivk and F. R. Le Diberder, Nucl. Instrum. and Methods Phys. Res. Sect A 555, 356 (2005).

[10] B. Aubert et al. [BaBar Collaboration], Phys. Rev. Lett. 96, 232001 (2006).

[11] A. Sokolov et al. [Belle Collaboration], Phys. Rev. D 79, 051103 (2009).

[12] A. Bondar et al. [Belle Collaboration], Phys. Rev. Lett. 108, 122001 (2012).

[13] A. Garmash et al. [Belle Collaboration], Phys. Rev. D 91, 072003 (2015).

[14] Y. H. Chen et al., Phys. Rev. D 95, 034022 (2017).

[15] Q. He et al. [CLEO Collaboration], Phys. Rev. Lett. 101, 192001 (2008).

[16] J. P. Lees et al. [BaBar Collaboration], Phys. Rev. D 84, 092003 (2011).

[17] U. Tamponi et al. [Belle Collaboration], Phys. Rev. D 87, no. 1, 011104 (2013).

[18] U. Tamponi et al. [Belle Collaboration], Phys. Rev. Lett. 115, 142001 (2015).

[19] F. K. Guo, C. Hanhart and U. G. Meissner, Phys. Rev. Lett. 105, 162001 (2010).

[20] K. F. Chen et al. [Belle Collaboration], Phys. Rev. Lett. 100, 112001 (2008).

[21] I. Adachi et al. [Belle Collaboration], Phys. Rev. Lett. 108, 032001 (2012).

[22] Preliminary result shown by P. Krokovny at the conference LaThuile 2012.

[23] Preliminary result shown by U. Tamponi at the conference DIS 2014.

[24] B. Wang, X. Liu and D. Y. Chen, Phys. Rev. D 94, no. 9, 094039 (2016). 